Национальный медицинский исследовательский центр эндокринологии, Москва

ОБОСНОВАНИЕ. Синдром диабетической стопы (СДС) - опасное осложнение сахарного диабета (СД), которое может приводить к развитию хронических ран и ампутациям. При наличии ран, резистентных к стандартным методам лечения, в качестве адъювантного метода лечения может использоваться рекомбинантный человеческий эпидермальный фактор роста (рчЭФР). В исследованиях продемонстрирована его клиническая эффективность, однако в литературных источниках представлено недостаточно информации об отдаленных результатах лечения, его безопасности и влиянии на прогрессирование осложнений СД, неблагоприятных сердечно-сосудистых событий и развитие онкологических заболеваний.

ЦЕЛЬ. Оценить отдаленные результаты терапии рчЭФР трофических язв стоп у лиц с множественными осложнениями СД.

МЕТОДЫ. В исследование были включены 20 пациентов с СД 1 и 2 типа (СД1 и СД2) и различными формами СДС без критической ишемии, которым ранее проводилось лечение СДС с использованием рчЭФР с целью оценки общего состояния, прогрессирования микро- и макрососудистых осложнений СД, неблагоприятных сердечно-сосудистых событий, развития онкологических заболеваний и качества жизни.

РЕзУЛЬТАТЫ. Выявлено статистически значимое различие между площадью раневых дефектов, процентом грануляционной ткани, заполнявшей раневой дефект, до начала лечения рчЭФР и на момент выписки из стационара (р<0,05). Во время лечения рчЭФР легкие нежелательные явления отмечались у $35 \%$ пациентов. Полная эпителизация ран у большинства пациентов наступила за 3 [2; 4] месяца. У 11,76\% пациентов рана полностью не эпителизировалась. Рецидив возник у 5,8\% в связи с несоблюдением разгрузки конечности. Малая ампутация была проведена 1 пациенту. У 23,5\% исследуемых выявлено прогрессирование диабетической ретино- и нефропатии. 11,76\% перенесли инфаркт миокарда (ИМ) неизвестной давности, 1 пациент (5,88\%) перенес острое нарушение мозгового кровообращения (ОНМК). Серьезные нежелательные явления в виде тромбоэмболии легочной артерии с летальным исходом и критической ишемии нижней конечности зафиксированы у $5,8 \%$.

ЗАКЛЮчЕНИЕ. В результате исследования отдаленных результатов лечения рчЭФР хронических ран стоп зафиксированы низкий процент рецидивов и малых ампутаций, отсутствие высоких ампутаций и онкологических заболеваний, развитие серьезных нежелательных явлений у 2 пациентов, прогрессирование диабетической ретинои нефропатии у 4 пациентов, развитие ИМ неизвестной давности у 2 пациентов и ОНМК у 1 пациента после терапии рчЭФР.

КЛЮЧЕВЫЕ СЛОВА: сахарный диабет; синдром диабетической стопы; эпидермальный фактор роста; трофические язвы; хронические раны

\title{
LONG-TERM FOLLOW-UP OF TREATMENT OF CHRONIC FOOT WOUNDS WITH RECOMBINANT HUMAN EPIDERMAL GROWTH FACTOR IN PATIENTS WITH DIFFERENT COMPLICATIONS OF DIABETES MELLITUS
}

\author{
(c) Ekaterina L. Zaitseva*, Viktor M. Zhilyaev, Gagik R. Galstyan
}

Endocrinology Research Centre, Moscow, Russia

BACKGROUND: Diabetic foot ulcer (DFU) is a dangerous complication of diabetes mellitus (DM), which can lead to the development of chronic wounds and amputations. Recombinant human epidermal growth factor (rhEGF) can be used as an adjuvant treatment for chronic wounds resistant to standard treatment. Studies have demonstrated its clinical efficacy, however, there is insufficient information on the long-term results of treatment, its safety and the effect on the progression of diabetes complications, adverse cardiovascular events and the development of cancer.

AIM: To assess the long-term results of rhEGF therapy for trophic foot ulcers in individuals with multiple complications of diabetes. 
METHODS: The study included 20 patients with type 1 and type 2 diabetes and various forms of DFS without critical ischemia, who had previously been treated with DFS using rhEGF in order to assess the general condition, progression of microand macrovascular complications of diabetes, adverse cardiovascular events, the development of cancer and the quality of life.

RESULTS: There was a statistically significant difference between the area of wound defects, the percentage of granulation tissue that filled the wound defect, before the start of rhEGF treatment and at the time of discharge from the hospital $(p<0.05)$. During treatment with rhEGF, mild adverse events were observed in $35 \%$. Complete epithelialization of wounds in most patients occurred in $3[2 ; 4]$ months. In 11.76\%, the wound was not completely epithelialized. Relapse occurred in $5.8 \%$ due to non-compliance with limb unloading. Minor amputation was performed in 1 patient. Progression of diabetic retino- and nephropathy was revealed in 23.5\%. 11.76\% suffered myocardial infarction of unknown age, 1 patient (5.88\%) suffered acute cerebrovascular accident. Serious adverse events in the form of PE with a fatal outcome and critical ischemia of the lower limb were recorded in $5.8 \%$.

CONCLUSIONS: As a result of the study of long-term results of rhEGF treatment of chronic foot wounds, a low percentage of relapses and small amputations, the absence of high amputations and oncological diseases, the development of serious adverse events in 2 patients, the progression of diabetic retino- and nephropathy in 4 patients, the development of IM of unknown age in 2 patients was recorded. and stroke in 1 patient after rhEGF therapy.

KEYWORDS: diabetes mellitus; diabetic foot ulcer; epidermal growth factor; diabetic foot ulcer; chronic wounds

Сахарный диабет (СД) представляет собой серьезную социально-экономическую проблему для глобального здравоохранения. На 2019 г. в мире насчитывалось 463 млн взрослых больных СД, по данным Международной федерации диабета. По оценкам специалистов, к 2030 г. эта цифра возрастет до 578,4 млн. Плохой контроль заболевания может приводить к серьезным и опасным для жизни осложнениям [1].

Синдром диабетической стопы (СДС) - осложнение СД, представляющее непосредственную угрозу развития язвенно-некротических процессов и гангрены стопы [2]. Распространенность язв на стопе у лиц с диагностированным СД составляет от 4 до 10\%, а вероятность развития данного осложнения в течение жизни может достигать 25\% [3]. Риск нетравматических ампутаций нижних конечностей у людей с СД по сравнению со здоровыми увеличивается в 15-20 раз. При этом большинству случаев ампутаций при диабете (до 85\%) предшествует плохо заживающая язва на стопе [4, 5]. Стандарт лечения СДС включает разгрузку пораженной конечности, восстановление артериального кровотока, лечение инфекции, компенсацию углеводного обмена и сопутствующих заболеваний, местное лечение ран (регулярный контроль состояния раны, очистка раны, в том числе с помощью скальпеля, выбор перевязочного средства, обеспечивающего контроль экссудации и влажной среды в ране, использование локального отрицательного давления для лечения послеоперационных ран), обучение пациентов правилам ухода за ногами, профилактику рецидивов [6]. При резистентности раневых дефектов нижних конечностей у лиц с СД к стандартному лечению могут применяться адъювантные методы, одним из которых является внутрираневое введение рекомбинантного человеческого эпидермального фактора роста (рчЭФР).

Эпидермальный фактор роста (ЭФР) был первым описанным фактором роста, выделенным из поднижнечелюстной слюнной железы мышей в 1962 г. [7]. ЭФР стимулирует миграцию фибробластов, эндотелиальных клеток в область язвы, формирование грануляционной ткани, ангиогенез, сокращение размеров раны с активацией миофибробластов, миграцию и пролиферацию эпителиальных клеток $[8,9]$.
Falanga в своем исследовании показал резкое снижение синтеза коллагена и замедление заживления [10]. При исследовании хронической раневой жидкости выявлено существенное разрушение добавленного ЭФР, а также рецептора ЭФР. Разрушение ЭФР и его рецептора было блокировано при добавлении ингибиторов металлопротеаз [11].

Tsang и соавт. в 2000-2002 гг. провели рандомизированное двойное слепое контролируемое исследование, которое продемонстрировало, что применение мази, содержащей человеческий ЭФР, в дополнение к стандартному лечению значительно сокращало время заживления диабетической язвы. Однако исследование было ограничено небольшим размером язв (I и II степень по Wagner) у испытуемых [12]. Все эти исследования способствовали поиску более эффективных стратегий лечения и способа применения для обеспечения активности ЭФР.

В итоге была сформирована идея о том, что инъекция ЭФР глубоко в основание и контуры раны позволила бы повысить фармакодинамический ответ с точки зрения роста грануляционной ткани и закрытия раны. На Кубе был создан препарат рчЭФР - Эберпрот-П (Heber Biotec, Гавана, Куба) - лекарственное средство, предназначенное для местного применения при лечении диабетических язв стопы. Препарат получают из штамма дрожжей Saccharomyces cerevisiae, в геном которого методами генной инженерии введен ген рчЭФР. Кубинские авторы постулируют тезис о том, что применение рчЭФР при СДС способствует более активному заживлению, снижает количество «высоких» ампутаций и способствует увеличению продолжительности жизни и ее качества [13].

Благодаря проведенному на Кубе в 2001-2002 гг. исследованию на пациентах с раневыми дефектами III-IV степени по Wagner, появились первые клинические данные об эффективном использовании внутрираневых инъекций рчЭФР при диабетических язвах стопы [14].

Вышеописанное исследование подтолкнуло к проведению рандомизированного двойного слепого клинического испытания II фазы, в котором была доказана эффективность внутрираневого введения 25 и 75 мкг 
рчЭФР в дополнение к стандартному лечению диабетических язв стопы [15]. Однако эти исследования были проведены на небольшой выборке и не были плацебо-контролируемыми. Чтобы подтвердить предыдущие выводы, было проведено многоцентровое рандомизированное плацебо-контролируемое исследование, которое подтвердило, что лечение внутрираневыми инъекциями рчЭФР в дополнение к стандартным методам по уходу за раной может оказать положительное влияние на исходы у пациентов с хроническими диабетическими язвами стопы. Полное закрытие раны произошло у 41 пациента (77,4\%) в группе, где применялась доза 75 мкг препарата, у 25 пациентов $(52,1 \%)$ в группе, где применялась доза 25 мкг, по сравнению с 27 пациентами $(56,2 \%)$ из группы плацебо [16]. Побочные эффекты были преимущественно легкими и умеренными (65,6\% - легкие, 28,6\% - умеренные и 3,7\% - тяжелые). Наиболее частыми нежелательными явлениями были боль и жжение в месте введения [17]. Вышеописанные исследования доказали эффективность применения данного препарата, характеризующуюся сокращением времени до полного заполнения язвенного дефекта грануляционной тканью, до его полной эпителизации, и снижением частоты ампутаций.

По результатам исследования, проведенного турецкими авторами, у пациентов с СДС, получавших стандартное лечение, дополнительное внутрираневое введение рчЭФР обеспечивало высокие темпы заживления с низкими показателями частоты случаев ампутации [18].

В 2019 г. опубликовано первое исследование, в котором сообщалось о долгосрочных результатах применения ЭФР у пациентов с СДС [19]. Наблюдение за 29 пациентами проводилось в течение 5 лет и показало низкую частоту рецидивов раневых дефектов и малых ампутаций, отсутствие высоких ампутаций.

Опубликованы данные о влиянии различных факторов роста на прогрессирование диабетической ретинопатии [20]. Основная роль принадлежит фактору роста эндотелия сосудов. Однако также имеются сведения об участии ЭФР в данном процессе [21].

В имеющихся литературных источниках мало информации об отдаленных результатах лечения препаратом рчЭФР - Эберпрот-П, отсутствуют данные о его влиянии на прогрессирование микро- и макрососудистых осложнений диабета, неблагоприятных сердечно-сосудистых событий, онкологических заболеваний, что и послужило предпосылкой для проведения данного исследования.

\section{ЦЕЛЬ}

Оценить отдаленные результаты терапии рчЭФР ран стоп у лиц с множественными микро- и макрососудистыми осложнениями СД.

\section{МЕТОДЫ}

\section{Дизайн исследования}

Исследование выполнено в дизайне обсервационного одноцентрового одномоментного неконтролируемого. Схема дизайна исследования представлена ниже.

\section{Критерии соответствия}

В проспективный этап исследования включены пациенты, соответствующие критериям включения и подписавшие добровольное информированное согласие.

Критерии включения:

1. возраст от 18 до 80 лет;

2. СД 1 типа (СД1) или СД 2 типа (СД2);

3. диагностированный ранее СДС, для лечения которого наряду со стандартными методами применялся препарат рчЭФР (Эберпрот П).

Критерий невключения:

1. отказ пациента от участия в исследовании.

\section{Условия проведения}

Исследование проведено в отделении диабетической стопы ФГБУ «НМИЦ эндокринологии» МЗ РФ (зав. отделением - проф. Галстян Г.Р.).

\section{Продолжительность исследования}

Исследование проводилось с июля 2019 г. по июнь 2020 г.

\section{Описание медицинского вмешательства}

В ходе анализа медицинской документации были оценены: состояние пациентов до госпитализации (выраженность поздних микро- и макрососудистых осложнений СД, наличие сердечно-сосудистых событий в анамнезе), форма (нейропатическая, нейро-ишемическая) и длительность СДС, размеры раневого дефекта до лечения, процент грануляционной ткани до лечения, наличие или отсутствие нежелательных явлений во время лечения препаратом рчЭФР (Эберпрот П) - головная боль, тремор, боль в месте введения, чувство жжения в месте инъекции, озноб, повышение температуры тела, инфекция в месте введения, эффективность препарата - динамика размера раны, процент грануляционной ткани при выписке из стационара.

Обследование включало в себя осмотр, анализ клинико-анамнестических данных, результатов лабораторно-инструментальных исследований (общеклинический анализ крови, биохимический анализ крови, определение уровня гликированного гемоглобина, рентгенография стопы в одной проекции, рентгенография легких, электрокардиография, эзофагогастродуоденоскопия).

Анализ качества жизни (КЖ) проведен с помощью опросника SF-36.

\section{Основной исход исследования}

Была собрана детальная информация о влиянии лечения рчЭФР пациентов с различными формами СДС на отдаленные события: процент рецидивов ран, количества малых ампутаций, серьезных нежелательных явлений (летальный исход, онкологические заболевания, высокая ампутация нижней конечности), прогрессирование поздних микро- и макрососудистых осложнений, частоту и характер основных неблагоприятных сердечно-сосудистых событий (инфаркт миокарда (ИМ), острые нарушения мозгового кровообращения (ОНМК)).

\section{Дополнительные исходы исследования}

В качестве дополнительного исхода исследования была проведена оценка КЖ с помощью опросника SF-36. 
Анализ в подгруппах

Были сформированы 2 подгруппы: пациенты, проанализированные ретроспективно, и пациенты, прошедшие очный осмотр.

\section{Методы регистрации исходов}

В ходе опроса пациентов и анализа медицинской документации оценены события, произошедшие за время после окончания госпитализации, в ходе которой пациенты получали терапию рчЭФР, и на момент включения в исследование. Оценено время, за которое рана полностью эпителизировалась, количество рецидивов ран в рамках СДС, число высоких ампутаций и малых операций на нижних конечностях, темпы прогрессирования поздних микро- и макрососудистых осложнений СД, частота неблагоприятных сердечно-сосудистых событий (ИМ, ОНМК), а также изучены данные анамнеза в отношении развития онкологических заболеваний.

Осмотр эндокринологом в кабинете диабетической стопы состоял из сбора анамнеза, осмотра стоп, исследования периферического кровотока с помощью портативного ультразвукового аппарата на тыльной и задней большеберцовой артериях стоп, оценки состояния тактильной чувствительности с помощью монофиламента массой 10 г, вибрационной чувствительности с помощью градуированного камертона (128 Гц) на бугре большеберцовой кости, медиальной лодыжке и на медиальной поверхности головки І-й плюсневой кости, температурной чувствительности с помощью прибора «Тип-Терм». Офтальмологическое обследование состояло из сбора анамнеза, осмотра век, определения подвижности глазного яблока, тонометрии с помощью бесконтактного тонометра ТГДц-01, офтальмобиомикроскопии переднего отрезка глаза с помощью стационарной щелевой лампы, прямой и обратной офтальмоскопии при помощи прямого и бинокулярного налобного офтальмоскопов.

Лабораторные тесты проведены по стандартным методикам в лаборатории «НМИЦ эндокринологии». В общеклиническом анализе крови оценивались уровень лейкоцитов с лейкоцитарной формулой (нейтрофилы, лимфоциты, моноциты, эозинофилы, базофилы), эритроцитов, гемоглобина, гематокрита, средний объем эритроцитов (MCV), среднее содержание гемоглобина в эритроците (MCH), средняя концентрация гемоглобина в эритроците (МСНC), индекс распределения эритроцитов (RDW), уровень тромбоцитов, средний объем тромбоцитов (MPV), тромбокрит (РСТ), индекс распределения тромбоцитов (PDW), скорость оседания эритроцитов (СОЭ). В биохимическом анализе крови оценивались липидный профиль (холестерин общий, липопротеиды низкой и высокой плотности, триглицериды), содержание креатинина, мочевины, мочевой кислоты, электролитов - натрия, калия, хлора, сывороточного железа, общего белка крови, аланинаминотрансферазы, аспартатаминотрансферазы. Для оценки выделительной способности почек проведен подсчет скорости клубочковой фильтрации (рСКФ) по формуле CKD-EPI. Также исследован уровень гликированного гемоглобина (анализатор BIO RAD D-10).

Инструментальные методы исследования включали в себя рентгенографию стопы в одной проекции, рентгенографию легких в прямой проекции на рентгенологи- ческом аппарате Optima RF 420, электрокардиографию с использованием электрокардиографа МАС 5500, эзофагогастродуоденоскопию с помощью гастроинтестинального видеоскопа Olympus GIF-XP 150N.

Другие методы. Проведен анализ КЖ пациентов с помощью наиболее широко распространенного опросника SF-36 [22]. Перевод на русский язык и апробация методики были проведены Институтом клинико-фармакологических исследований (Санкт-Петербург). 36 пунктов опросника сгруппированы в восемь шкал. Показатели каждой шкалы варьируют между 0 и 100, где 100 представляет полное здоровье, все шкалы формируют два показателя: душевное и физическое благополучие. Результаты представляются в виде оценок в баллах по 8 шкалам.

1. Physical Functioning (PF) - физическое функционирование, отражающее степень, в которой физическое состояние ограничивает выполнение физических нагрузок (самообслуживание, ходьба, подъем по лестнице, переноска тяжестей и т.п.).

2. Role-Physical Functioning (RP) - ролевое функционирование, обусловленное физическим состоянием, влияние физического состояния на повседневную ролевую деятельность (работу, выполнение повседневных обязанностей).

3. Bodily pain (BP) - интенсивность боли и ее влияние на способность заниматься повседневной деятельностью, включая работу по дому и вне дома.

4. General Health (GH) - общее состояние здоровья оценка больным своего состояния здоровья в настоящий момент и перспектив лечения.

5. Vitality (VT) - жизнеспособность (подразумевает ощущение себя полным сил и энергии или, напротив, обессиленным).

6. Social Functioning (SF) - социальное функционирование, определяется степенью, в которой физическое или эмоциональное состояние ограничивает социальную активность (общение).

7. Role-Emotional (RE) - ролевое функционирование, обусловленное эмоциональным состоянием, - влияние эмоционального состояния на ролевое функционирование предполагает оценку степени, в которой эмоциональное состояние мешает выполнению работы или другой повседневной деятельности (включая увеличение затрат времени, уменьшение объема выполненной работы, снижение качества ее выполнения и т. п.).

8. Mental Health (MH) - психическое здоровье - самооценка психического здоровья, характеризует настроение (наличие депрессии, тревоги, общий показатель положительных эмоций).

Опрос производился следующим способом: самостоятельное заполнение пациентом анкеты SF-36. Для произведения сложных математических расчетов была использована компьютерная модель опросника SF-36 Test SF-36 by JR ver.1.2. С ее помощью были рассчитаны основные показатели КЖ. Затем проводилась стандартизация шкал в соответствии с протоколом, использованным в многоцентровом исследовании КЖ «МИРАЖ» [23].

Этическая экспертиза

Локальный этический комитет при ФГБУ «НМИЦ эндокринологии» Минздрава России постановил одобрить 
возможность проведения данной научно-исследовательской работы, выписка из протокола № 13 от 4 сентября 2019 г. Всеми пациентами подписаны информированные согласия на участие в исследовании.

\section{Статистический анализ}

Принципы расчета размера выборки. Размер выборки предварительно не рассчитывался.

Методы статистического анализа данных. Для анализа результатов исследования использовалась программа Statistica 7.0 (TIBCO Software Inc., США). Описание количественных данных представлено в виде медианы (Ме) и квартилей (25 и 75 перцентили, Q25-75). Для описания качественных данных рассчитывали абсолютные (n) и относительные значения (\%). Для анализа вида распределений применялись критерии Шапиро-Уилка. Сравнение независимых групп по количественным признакам осуществлялось непараметрическим методом с использованием критерия Вилкоксона. Статистически значимыми считали различия при $\mathrm{p}<0,05$.

\section{РЕЗУЛЬТАТЫ}

Объекты (участники) исследования

В исследование были включены 20 пациентов с СД1 и СД2, множественными микро- и макрососудистыми осложнениями, получивших более 2 лет назад лечение инъекциями в область раны препарата рчЭФР (Эберпрот П).

\section{Основные результаты исследования}

Был проведен анализ архивных карт пациентов, которым проводилось местное лечение рчЭФР. Исходное соматическое состояние пациентов до начала терапии рчЭФР указано в табл. 1.

Во время лечения рчЭФР легкие нежелательные явления отмечались у 7 пациентов (35\%): боль в месте инъекции - 5, озноб - 2, однократная рвота, жидкий стул - 1. Серьезных нежелательных явлений за время лечения не зафиксировано. Прервать терапию рчЭФР пришлось у 1 пациента из-за появления жалоб на рвоту, жидкий стул.

Таблица 1. Клиническая характеристика пациентов $(\mathrm{n}=20)$

\begin{tabular}{|c|c|}
\hline Показатель & $(n=20)$ \\
\hline Возраст, лет & $60[53,5 ; 68]$ \\
\hline Пол, м/ж & $13 / 7$ \\
\hline Тип СД, 1/2 & $4 / 16$ \\
\hline Длительность течения СД, лет & $16,5[9 ; 19]$ \\
\hline Количество инъекций рчЭФР (n) & $6[5 ; 6]$ \\
\hline \multicolumn{2}{|l|}{ Дистальная диабетическая полинейропатия (n): } \\
\hline нет & 0 \\
\hline есть & 20 \\
\hline \multicolumn{2}{|l|}{ Форма СДС (n): } \\
\hline нейропатическая & 11 \\
\hline нейроишемическая & 9 \\
\hline Длительность СДС до лечения, недели & $6,5[5 ; 10]$ \\
\hline \multicolumn{2}{|l|}{ Степень раневых дефектов по Вагнеру (n): } \\
\hline II & 3 \\
\hline III & 17 \\
\hline \multicolumn{2}{|l|}{ Площадь раневых дефектов, см²: } \\
\hline до лечения & $8,75[4 ; 15]$ \\
\hline на момент выписки из стационара & $3,5[1,8 ; 6,15]$ \\
\hline \multicolumn{2}{|l|}{ Грануляционная ткань, выстилающая дно раны, \%: } \\
\hline до лечения & $12,5[0 ; 20]$ \\
\hline на момент выписки из стационара & $95[90 ; 100]$ \\
\hline \multicolumn{2}{|l|}{ Диабетическая нефропатия, ХБП, стадия (n): } \\
\hline $\mathrm{C} 1$ & 15 \\
\hline $\mathrm{C} 2$ & 3 \\
\hline $\mathrm{C} 3 \mathrm{a}$ & 1 \\
\hline C36 & 0 \\
\hline C4 & 0 \\
\hline $\mathrm{C} 5$ & 1 \\
\hline \multicolumn{2}{|l|}{ Сердечно-сосудистые осложнения в анамнезе (n): } \\
\hline Отсутствует & 12 \\
\hline Инфаркт миокарда & 6 \\
\hline Инсульт & 2 \\
\hline
\end{tabular}

Примечания: СД - сахарный диабет; рчЭФР — рекомбинантный человеческий эпидермальный фактор роста; СДС — синдром диабетической стопы; ХБП - хроническая болезнь почек; 


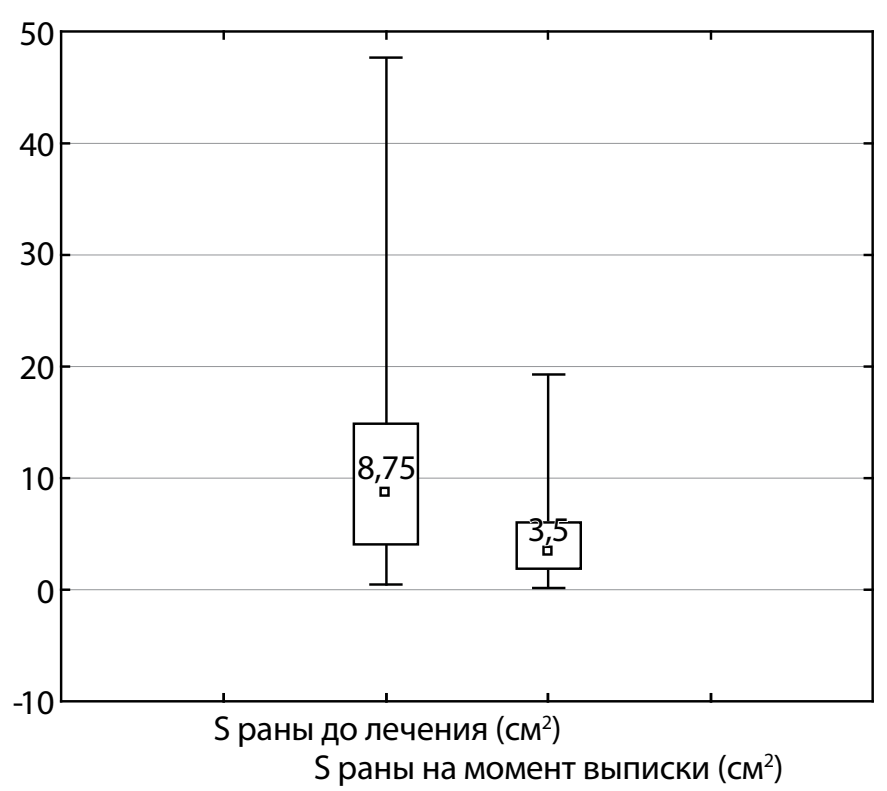

- Median \25-75\% I Min-Max

Рисунок 1. Площадь раны до лечения рекомбинантным человеческим эпидермальным фактором роста и на момент выписки из стационара.

В ходе анализа было выявлено статистически значимое различие между площадью раневых дефектов, количеством грануляционной ткани до начала лечения рчЭФР и на момент выписки из стационара $(p<0,05)$. Медиана площади раневых дефектов до лечения составила $8,75 \mathrm{~cm}^{2}[4 ; 15]$, на момент выписки - 3,5 см² $[1,8 ; 6,15]$. Медиана количества грануляционной ткани составила до лечения 12,5\% [0; 20], на момент выписки 95\% [90; 100]. Результаты представлены на рис. 1 и 2.

На очный осмотр в центр явились 17 пациентов, 1 пациент умер, 2 отказались приехать.

Клинико-лабораторная характеристика пациентов представлена в табл. 2.

Полная эпителизация раны у большинства пациентов наступила за 3,5 [2,5; 4] месяца. У 2 из 17 пациентов $(11,76 \%)$ раневой дефект полностью не эпителизировался. Рецидив возник у 1 пациента (5,88\%) через 2 недели после полной эпителизации в связи с несоблюдением разгрузки нижней конечности.

Малая ампутация была проведена у 1 пациента (5,88\%). Среди обследованных пациентов никому не проводилось высоких ампутаций. Серьезные нежелательные явления зафиксированы в 2 случаях из 20 (10\%) смерть 1 пациента, причина - тромбоэмболия легочной артерии (ТЭЛА), также у 1 пациента развилась критическая ишемия правой нижней конечности, потребовавшая проведения реваскуляризации.

Проведена оценка прогрессирования поздних микро- и макрососудистых осложнений СД: у 4 пациентов (23,5\%) выявлено прогрессирование нефропатии у 1 пациента до ХБП С 5, у 3 пациентов до ХБП СЗа. У 4 пациентов (23,5\%) выявлено прогрессирование диабетической ретинопатии до пролиферативной стадии. У данных пациентов уровень гликированного гемоглобина в среднем составлял $9,7 \%$.

В ходе исследования также проведена оценка частоты и характера основных неблагоприятных сердечно-сосудистых событий: 2 пациента (11,76\%) перенесли

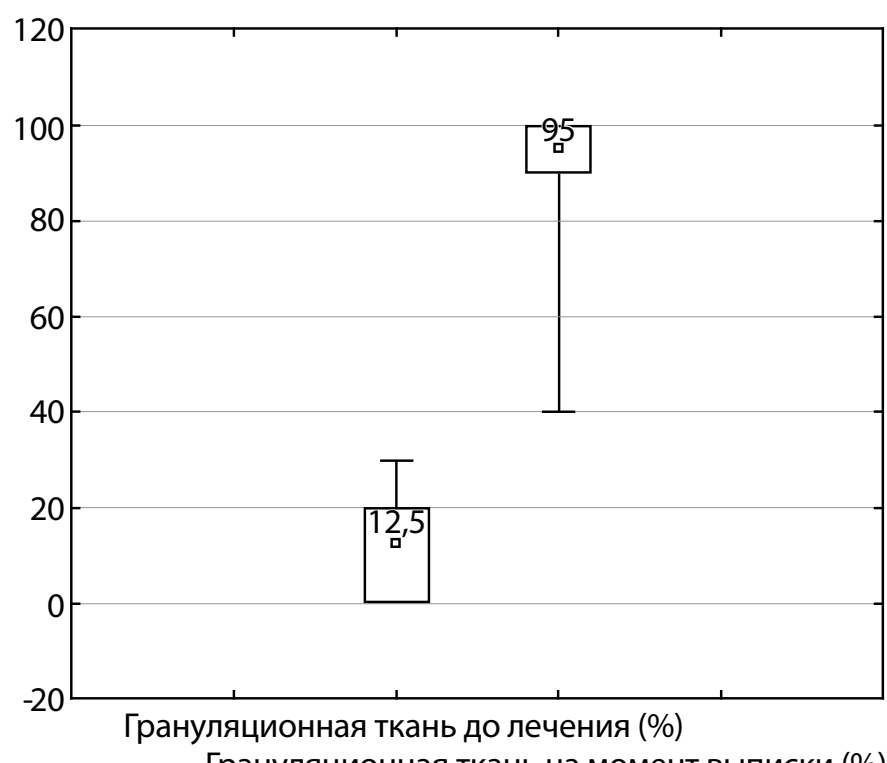

Грануляционная ткань на момент выписки (\%)

- Median $\square 25-75 \%$ T Min-Max

Рисунок 2. Процент грануляционной ткани до лечения рекомбинантным человеческим эпидермальным фактором роста и на момент выписки из стационара.

ИМ неизвестной давности, 1 пациент (5,88\%) перенес ОНМК.

Среди показателей общеклинического и биохимического анализов крови обращают на себя внимание низкий уровень хс-ЛПВП и высокий уровень хс-ЛПНП у исследуемых (см. табл. 2).

Таблица 2. Клинико-лабораторная характеристика пациентов, явившихся на контрольное обследование $(\mathrm{n}=17)$

\begin{tabular}{|c|c|}
\hline Показатель & $(n=17)$ \\
\hline Возраст, лет & $61[55 ; 66]$ \\
\hline Пол, м/ж & $11 / 6$ \\
\hline Тип СД (1/2) & $3 / 14$ \\
\hline $\mathrm{HbA}_{1 \mathrm{c}^{\prime}} \%$ & $8,7[7,2 ; 9,8]$ \\
\hline Гемоглобин, г/л & $139[125 ; 144]$ \\
\hline Лейкоциты, 109/л & $7,41[6,3 ; 9]$ \\
\hline $\operatorname{CO\ni }, \mathrm{mm} / 4$ & $30[16 ; 42]$ \\
\hline хс-ЛПНП, моль/л & $2,4[1,5 ; 3,2]$ \\
\hline ТГ, ммоль/л & $1,8[1,4 ; 2,76]$ \\
\hline хс-ЛПВП, ммоль/л & $1,0[0,8 ; 1,3]$ \\
\hline рСКФ (ЕРІ), мл/мин/1,73 м² & $63,5[57 ; 85,8]$ \\
\hline Белок общ. сыворотки, г/л & $75[72 ; 76]$ \\
\hline Мочевая кислота, мкмоль/л & $313[288,6 ; 376]$ \\
\hline Железо сывороточное, мкмоль/л & $13,4[9,5 ; 17]$ \\
\hline АЛТ, Ед/л & $21[11 ; 26]$ \\
\hline АСТ, Ед/л & $18[16 ; 20]$ \\
\hline Калий, ммоль/л & $4,7[4,5 ; 4,9]$ \\
\hline
\end{tabular}

Примечания: СОЭ - скорость оседания эритроцитов, хс-ЛПНП холестерин липопротеинов низкой плотности; ТГ - триглицериды; хс-ЛПВП - холестерин липопротеинов высокой плотности; СКФ скорость клубочковой фильтрации; АЛТ - аланинаминотрансфераза; АСТ - аспартатаминотрансфераза. 
В ходе анамнестических сведений, анализа медицинской документации и проведенного онкопоиска (эзофагогастродуоденоскопия (проведена 15 пациентам, 2 отказались), рентгенография легких (проведена всем пациентам)) данных за наличие онкологических заболеваний у обследованных пациентов не получено.

\section{Дополнительные результаты исследования}

Проведен анализ КЖ пациентов с помощью опросника SF-36. Показатели стандартизированных 8 шкал КЖ опросника SF-36 представлены в табл. 3.

\section{Нежелательные явления}

В ходе исследования нежелательных явлений зафиксировано не было.

\section{ОБСУЖДЕНИЕ}

\section{Резюме основного результата исследования}

В проведенном исследовании отмечался положительный эффект лечения рчЭФР в виде сокращения размеров раневых дефектов, увеличения процента грануляционной ткани, заполняющей дно раны, зафиксировано небольшое количество рецидивов и малых ампутаций в ходе проспективного наблюдения. Также не получено данных за развитие злокачественных образований. С другой стороны, в ходе проспективного этапа у части пациентов выявлено прогрессирование диабетических ретино- и нефропатии, развитие серьезных нежелательных явлений - критическая ишемия нижней конечности у 1 пациента и смерть 1 пациента в результате тромбоэмболии легочной артерии. При анализе основных неблагоприятных сердечно-сосудистых событий: у 2 пациентов развился ИМ, у 1 пациента — OHMК.

\section{Обсуждение основного результата исследования}

В ходе проведенного исследования показана эффективность препарата рчЭФР - выявлены клинически значимые различия в размере раневых дефектов и проценте грануляционной ткани до и после лечения. Частота нежелательных явлений оказалась ниже, чем в исследованиях предыдущих авторов $[17,18]$, что может быть связано с малым размером выборки. Обращают на себя внимание большое число пациентов, достигших полной эпителизации раневых дефектов, а также низкий показатель рецидивов.

Определен также процент прогрессирования поздних микро- и макрососудистых осложнений. Особенно обращает на себя внимание прогрессирование диабетических ретино- и нефропатии. Имеются данные об участии ЭФР в прогрессировании диабетической ретинопатии [21]. Характерно то, что у данных пациентов выявлен уровень гликированного гемоглобина, значительно превышающий индивидуальные целевые значения, что может являться одной из причин прогрессирования вышеуказанных осложнений СД. Данное обстоятельство затрудняет установление связи прогрессирования осложнений СД с лечением рчЭФР, что требует дальнейшего изучения.

В исследовании не получено данных о развитии злокачественных новообразований, что согласуется с информацией о том, что ЭФР, в отличие от некоторых членов его семейства, не оказывает влияния на онкологические риски [24].

Оценивая результаты по каждой шкале КЖ, учитывалось то, что показатель 100 представляет собой полное здоровье, а 0 - его отсутствие. Показатели физического функционирования (PF) и общего состояния здоровья $(\mathrm{GH})$ пациентов находятся на достаточно низком уровне, что может быть связано с наличием у части респондентов ампутированных пальцев, большого количества заболеваний, ограничивающих самообслуживание, ходьбу, подъем по лестнице, переноску тяжестей и т.п. Полученные показатели ролевого функционирования, обусловленного физическим состоянием (RP), интенсивности боли (BP), жизнеспособности (VT) и психического здоровья (MH) не оказывают значимого влияния на КЖ пациентов, так как находятся в середине отрезка. Высокие показатели шкал социального функционирования (SF), ролевого функционирования, обусловленного эмоциональным состоянием (RE), могут говорить о том, что эмоциональное состояние не ограничивает социальную активность пациентов, не мешает выполнению работы или другой повседневной деятельности

\section{Ограничения исследования}

К существенным ограничениям исследования относится отсутствие группы контроля, а также небольшой объем выборки пациентов.

Достаточно сложно было обеспечить плацебо-контролируемое исследование в связи с трудностью подбора сопоставимых групп по течению раневых процессов.

Наиболее вероятной причиной прогрессирования микро- и макрососудистых осложнений СД являлся неудовлетворительный контроль диабета, что было подтверждено показателями гликированного гемоглобина.

В будущих исследованиях необходимо обеспечить более частые визиты пациентов на осмотр для оценки приверженности пациентов лечению и проведения своевременной коррекции сахароснижающей терапии, а также формирование контрольной группы.

Таблица 3. Результаты оценки качества жизни с помощью опросника SF-36

\begin{tabular}{lcccccccc}
\hline \multirow{2}{*}{$\begin{array}{c}\text { Средние значения, } \\
\text { процентили, размах }\end{array}$} & \multicolumn{9}{c}{ Шкалы SF-36 } \\
\cline { 2 - 9 } \multicolumn{1}{c}{} & $\mathbf{P F}$ & $\mathbf{R P}$ & $\mathbf{B P}$ & $\mathbf{G H}$ & $\mathbf{V T}$ & $\mathbf{S F}$ & $\mathbf{R E}$ & MH \\
\hline $25 \%$ & 33 & 37 & 42 & 37 & 43 & 47 & 44 & 44.5 \\
$50 \%$ (Me) & 37 & 49 & 47 & 38,8 & 50 & 57,6 & 60 & 53,5 \\
$75 \%$ & 49 & 61 & 54,8 & 51,7 & 54 & 62 & 60 & 58,6 \\
\hline
\end{tabular}

Примечания: PF — физическое функционирование; RP — ролевое функционирование, обусловленное физическим состоянием; ВP — интенсивность боли; GH - общее состояние здоровья; VT — жизненная активность; SF — социальное функционирование; RE — ролевое функционирование, обусловленное эмоциональным состоянием; МH - психическое здоровье. 
Учитывая то, что не проводилась оценка КЖ у пациентов до лечения рчЭФР, а также отсутствие контрольной группы, не представляется возможным провести сравнение полученных результатов.

\section{ЗАКЛЮЧЕНИЕ}

Данное исследование является первой опубликованной работой, посвященной изучению отдаленных результатов лечения хронических ран нижних конечностей рчЭФР у пациентов с множественными микрои макрососудистыми осложнениями СД в Российской Федерации. В ходе исследования зафиксированы: низкий процент рецидивов ран нижних конечностей, малых ампутаций, отсутствие высоких ампутаций и онкологических заболеваний, развитие серьезных нежелательных явлений у 2 пациентов, прогрессирование диабетической ретинопатии у 4 пациентов и диабетической нефропатии у 4 пациентов, развитие ИМ у 2 пациентов и ОНМК у 1 пациента. Полученные данные имеют описательный характер, но в то же время позволяют рассмотреть отдаленное влияние лечения рчЭФР на состояние пациентов. Результаты настоящего исследования могут помочь в проведении будущих работ по данной теме.
В последующих исследованиях необходимо рассмотреть более длительный период наблюдения, включение группы контроля, а также больший размер выборки.

\section{ДОПОЛНИТЕЛЬНАЯ ИНФОРМАЦИЯ}

Источник финансирования. Исследование выполнено при финансовой поддержке научно-исследовательской работы в рамках гранта №МК-2200.2019.7 «Отдаленные результаты комплексного лечения хронических ран нижних конечностей у пациентов с множественными микро- и макрососудистыми осложнениями сахарного диабета с использованием рекомбинантного человеческого эпидермального фактора роста».

Конфликт интересов. Авторы декларируют отсутствие явных и потенциальных конфликтов интересов, связанных с публикацией настоящей статьи.

Участие авторов. Зайцева Е.Л. - сбор клинического материала, его анализ, опрос, осмотр пациентов написание статьи; Жиляев В.М. сбор клинического материала, его анализ, статистическая обработка данных, написание статьи; Галстян Г.Р. - написание статьи. Все авторы внесли значимый вклад в подготовку статьи, прочли и одобрили финальную версию статьи до публикации.

Благодарности. Авторы выражают благодарность всем сотрудникам отделения диабетической стопы ФГБУ «НМИЦ эндокринологии».

\section{СПИСОК ЛИТЕРАТУРЫ| REFERENCES}

1. IDF atlas (9th edition). [Internet]. Brussels, Belgium International Diabetes Federation; 2019. Available from: http://www.diabetesatlas.org

2. Алгоритмы специализированной медицинской помощи больным сахарным диабетом / Под редакцией И.И. Дедова, М.В. Шестаковой, А.Ю. Майорова. 9-й выпуск (дополненный) // Сахарный диабет. 2019. - T. 22. — №151. — C. 1-144. [Standards of specialized diabetes care. Edited by Dedov II, Shestakova MV, Mayorov AYu. 9th edition. Diabetes mellitus. 2019;22(151):1-144. (In Russ.)]. doi: https://doi.org/10.14341/DM221S1

3. Singh N. Preventing Foot Ulcers in Patients With Diabetes. JAMA. 2005;293(2):217. doi: https://doi.org/10.1001/jama.293.2.217

4. Pemayun TGD, Naibaho RM, Novitasari D, Amin N, Minuljo TT. Risk factors for lower extremity amputation in patients with diabetic foot ulcers: a hospital-based case-control study. Diabet Foot Ankle. 2015;6(1):29629. doi: https://doi.org/10.3402/dfa.v6.29629

5. Ramsey SD, Newton K, Blough D, et al. Incidence, outcomes, and cost of foot ulcers in patients with diabetes. Diabetes Care. 1999;22(3):382-387. doi: https://doi.org/10.2337/diacare.22.3.382

6. Jakosz N. Book review - IWGDF Guidelines on the Prevention and Management of Diabetic Foot Disease. Wound Pract Res. 2019;27(3):144. doi: https://doi.org/10.33235/wpr.27.3.144

7. Cohen S. Isolation of a Mouse Submaxillary Gland Protein Accelerating Incisor Eruption and Eyelid Opening in the New-born Animal. J Biol Chem. 1962;237(5):1555-1562. doi: https://doi.org/10.1016/S0021-9258(19)83739-0

8. Berlanga-Acosta J. Diabetic lower extremity wounds: the rationale for growth factors-based infiltration treatment. Int Wound J. 2011;8(6):612-620. doi: https://doi.org/10.1111/j.1742-481X.2011.00840.x

9. Gibbs S, Silva Pinto AN, Murli S, et al. Epidermal growth factor and keratinocyte growth factor differentially regulate epidermal migration, growth, and differentiation. Wound Repair Regen. 2000;8(3):192-203. doi: https://doi.org/10.1046/j.1524-475x.2000.00192.x

10. Falanga V. Growth Factors and Chronic Wounds: The Need to Understand the Microenvironment. J Dermatol. 1992;19(11):667-672. doi: https://doi.org/10.1111/j.1346-8138.1992.tb03756.x

11. Mast BA, Schultz GS. Interactions of cytokines, growth factors, and proteases in acute and chronic wounds. Wound Repair Regen. 1996;4(4):411-420. doi: https://doi.org/10.1046/j.1524-475X.1996.40404.x
12. Tsang MW, Wong WKR, Hung CS, et al. Human Epidermal Growth Factor Enhances Healing of Diabetic Foot Ulcers. Diabetes Care. 2003;26(6):1856-1861. doi: https://doi.org/10.2337/diacare.26.6.1856

13. Montequin JF, Bonachea LS, Acosta JB, et al. Intralesional and perilesional application of an epidermal growth factor (Heberprot-P®) in diabetic foot ulcers. Part one. Angiology and Vascular Surgery. 2018;24(4):33-42

14. Acosta JB, Savigne W, Valdez C, et al. Epidermal growth factor intralesional infiltrations can prevent amputation in patients with advanced diabetic foot wounds. Int Wound J. 2006;3(3):232-239. doi: https://doi.org/10.1111/j.1742-481X.2006.00237.x

15. Fernández-Montequín JI, Infante-Cristiá E, Valenzuela-Silva C, et al. Intralesional injections of Citoprot- $P$ ${ }^{*}$ (recombinant human epidermal growth factor) in advanced diabetic foot ulcers with risk of amputation. Int Wound J. October 2007:4(4):333-343. doi: https://doi.org/10.1111/j.1742-481X.2007.00344.x

16. Fernández-Montequín JI, Valenzuela-Silva CM, González-Díaz O, et al. Intra-lesional injections of recombinant human epidermal growth factor promote granulation and healing in advanced diabetic foot ulcers: multicenter, randomised, placebocontrolled, double-blind study. Int Wound J. 2009;6(6):432-443. doi: https://doi.org/10.1111/j.1742-481X.2009.00641.x

17. Fernández-Montequín Jl, Betancourt BY, Leyva-Gonzalez G, et al. Intralesional administration of epidermal growth factor-based formulation (Heberprot-P) in chronic diabetic foot ulcer: treatment up to complete wound closure. Int Wound J. 2009;6(1):67-72. doi: https://doi.org/10.1111/j.1742-481X.2008.00561.x

18. Ertugrul BM, Lipsky BA, Guvenc U. An Assessment of Intralesional Epidermal Growth Factor for Treating Diabetic Foot Wounds. J Am Podiatr Med Assoc. 2017;107(1):17-29. doi: https://doi.org/10.7547/15-056

19. Kahraman M, Misir A, Kizkapan TB, et al. The Long-Term Outcomes Following the Application of Intralesional Epidermal Growth Factor in Patients With Diabetic Foot Ulcers. J Foot Ankle Surg. 2019;58(2):282-287. doi: https://doi.org/10.1053/j.jfas.2018.08.041

20. Grant MB, Afzal A, Spoerri P, et al. The role of growth factors in the pathogenesis of diabetic retinopathy. Expert Opin Investig Drugs. 2004;13(10):1275-1293. doi: https://doi.org/10.1517/13543784.13.10.1275

21. Khan ZA, Chakrabarti S. Growth Factors in Proliferative Diabetic Retinopathy. Exp Diabesity Res. 2003;4(4):287-301. doi: https://doi.org/10.1155/EDR.2003.287 
22. Ware JE, Snow KK, Kosinski M, Gandek B. SF-36 Health Survey Manual and Interpretation Guide. Bost New Engl Med Cent. 1993.

23. Амирджанова В.Н., Горячев Д.В., Коршунов Н.И., и др. Популяционные показатели качества жизни по опроснику SF-36 // Научно-практическая ревматология. - 2008. №1. - C. 36-48. [Amirdjanova VN, Goryachev DV, Korshunov NI, et al. SF-36 questionnaire population quality of life indices Objective. Rheumatol Sci Pract. 2008;122(1):36-48 (In Russ.)]. doi: https://doi.org/10.14412/1995-4484-2008-852

24. Wilson KJ, Gilmore JL, Foley J, et al. Functional selectivity of EGF family peptide growth factors: Implications for cancer. Pharmacol Ther. 2009;122(1):1-8. doi: https://doi.org/10.1016/j.pharmthera.2008.11.008

\section{ИНФОРМАЦИЯ ОБ АВТОРАХ [AUTHORS INFO]}

*Зайцева Екатерина Леонидовна, к.м.н., С.н.с. [Ekaterina L. Zaitseva, MD, PhD, senior research associate]; aдpec: Россия, 117036, Москва, ул. Дм. Ульянова, д. 11 [address: 11 Dm. Ulyanova street, 117036 Moscow, Russian Federation]; ORCID: https://orcid.org/0000-0002-3735-019X; eLibrary SPIN: 1075-3022; e-mail: zai.kate@gmail.com

Жиляев Виктор Максимович, клинический ординатор [Viktor M. Zhilyaev, clinical residence]; ORCID: https://orcid.org/0000-0002-2137-9879; eLibrary SPIN: 7728-9212; e-mail: wolfzhvm@gmail.com Галстян Гагик Радикович, д.М.н., профессор [Gagik R. Galstyan, MD, PhD, Professor]; ORCID: https://orcid.org/0000-0001-6581-4521; eLibrary SPIN: 9815-7509; e-mail: galstyangagik964@gmail.com

\section{ЦИТИРОВАТЬ:}

Зайцева Е.Л., Жиляев В.М., Галстян Г.Р. Отдаленные результаты лечения хронических ран стоп рекомбинантным человеческим эпидермальным фактором роста у пациентов с осложнениями сахарного диабета // Сахарный диабет. 2020. — T. 23. — №6. - C. 532-540. doi: https://doi.org/10.14341/DM12701

\section{TO CITE THIS ARTICLE:}

Zaitseva EL, Zhilyaev VM, Galstyan GR. Long-term Follow-up of treatment of chronic foot wounds with recombinant human epidermal growth factor in patients with different complications of diabetes mellitus. Diabetes Mellitus. 2020;23(6):532-540. doi: https://doi.org/10.14341/DM12701 